\title{
Synthesis and Characterization of Stabilized Zirconia Toughened Alumina (ZTA) Doped Niobium Pentoxide (Nb2O5)
}

\author{
B. Johar ${ }^{1,}{ }^{*}, Z$. Zul. Azhar ${ }^{1}$, and Z.A. Ahmad ${ }^{2}$ \\ ${ }^{1}$ School of Materials Engineering, Universiti Malaysia Perlis, 02600 Kangar, Perlis, Malaysia \\ ${ }^{2}$ School of Materials and Mineral Resources Engineering, Universiti Sains Malaysia, \\ Engineering Campus, 14300 Nibong Tebal, Penang, Malaysia
}

\begin{abstract}
The main target of this research is to study the mechanical properties of the $\mathrm{Nb}_{2} \mathrm{O}_{5}$ doped with alumina-zirconia composites. Pellets form samples of ZTA (5YSZ) doped niobium pentoxide in varied amount of $0 \mathrm{wt} \%, 0.3 \mathrm{wt} \%, 0.5 \mathrm{wt} \%$ and $0.7 \mathrm{wt} \%$ were prepared by solid-state conventional method with respective $4: 1$ ratio or alumina and 5YSZ. The effect of different dopant addition on the morphology, phase transformation and hardness was studied. Each mixture of the compositions were calcined at $1000^{\circ} \mathrm{C}$ followed by pressing at $220 \mathrm{MPa}$ and then they were sintered at $1650^{\circ} \mathrm{C}$ with heating rate $10^{\circ} \mathrm{C} \min ^{-1}$ and holding for 2 hours in air. Phase transformation, phase quantification and crystal structure analysis was revealed by using X-ray diffraction data with the help of X-Pert HighScore Plus software using Rietveld Refinement Method while microstructure was observed by scanning electron microscopy. The hardness of the composite is determined by using Vickers hardness test. It found that with $0.5 \mathrm{wt} \%$ niobium pentoxide addition would give a great effect on the microstructure distribution, densification and mechanical properties of the ZTA-doped sample.
\end{abstract}

\section{Introduction}

Zirconia toughened ceramics have proven to give abrasion, wear resistance and they can be last longer up to 10 time compared to steel tool parts [1]. Zirconia toughened alumina is the composite that involves alumina matrix and zirconia particles as reinforcement where they are enhancing each other by embedded zirconia particle into alumina matrix [2-4]. This kind of ceramics composite includes the combination of hardness provided by alumina with fracture toughness and strength from zirconia particles.

Basically, zirconia wills presence in 3 crystallographic forms, which are monoclinic, tetragonal and cubic phases [2]. Zirconia is in the monoclinic phase at ambient temperature and in tetragonal phase when heat is applied up to $1700^{\circ} \mathrm{C}$. Furthermore, at about $2370{ }^{\circ} \mathrm{C}$, it is changes into a cubic phase [5]. The transformation of tetragonal to monoclinic phase will resulted in toughening mechanism of alumina zirconia composite materials and the system can be stabilized with the addition of some oxides materials in order to maintain the

* Corresponding author: banjuraizah@unimap.edu.my 
tetragonal phase in the ambient temperature $[2,6]$. This is because zirconia in tetragonal phase shows excellent mechanical properties. In addition, the transformation of tetragonal phase into monoclinic phase during cooling process will cause the volume increment that may lead to failure [5,6]. Failures also may cause by the presence of porosity, inhomogeneity in phase, grain size, micro-crack and any other failure possibilities which lowering the mechanical properties of the ceramics materials [7].

$\mathrm{Al}_{2} \mathrm{O}_{3}$ matrix reinforced with $\mathrm{ZrO}_{2}$ particles that are called as ZTA. According to Hannink et al., in their study showed that addition of the niobium pentoxide contents affect some properties such as microstructure, hardness, fracture toughness and densification of the composites [8]. Therefore, the main purpose of this research is to investigate the effect of the ZTA doped with niobium oxide which focusing on the hardness properties of alumina and zirconia ceramics materials.

Researchers have studied that the densification and mechanical properties of samples can be enhanced by the addition of $\mathrm{Nb}_{2} \mathrm{O}_{5}[6,12]$. The enhancement of ceramics composite material can be accomplished by using different type of manipulated dopant contents to improve their mechanical properties such as hardness and fracture toughness that prompted to various applications. According to Hassan et al. the addition of $0.2-0.6 \mathrm{vol} \%$ of $\mathrm{Nb}_{2} \mathrm{O}_{5}$ showed an increment in bending strength, hardness, relative density and reduce in porosity of the ceramics materials [2]. Addition of zirconia particles in the alumina matrix will result in the both high fracture toughness and hardness compared to pure alumina and zirconia ceramics materials [15]. All the factors described above lead to this research scope. In this research, $5 \mathrm{~mol} \% \mathrm{YSZ}$ and alumina with 1:4 ratio varies with the addition amount of $0 \mathrm{wt} \%$ to $0.7 \mathrm{wt} \%$ of $\mathrm{Nb}_{2} \mathrm{O}_{5}$ were prepared. The target of this research is to investigate the stabilized zirconia toughened alumina (ZTA) doped with different weight percent of niobium oxide that will affect the hardness, morphology and phase transformation of the specimen.

\section{Materials and methods}

\subsection{Materials}

The main raw materials used in the present research are alumina, $\mathrm{Al}_{2} \mathrm{O}_{3}$ (MAJU SaintifikSdn. Bhd., 99\% purity), 5YSZ, $\mathrm{ZrO}_{2}+5$ mol\% $\mathrm{Y}_{2} \mathrm{O}_{3}$ (MAJU SaintifikSdn. Bhd., purity of $96 \%$ ) and niobium pentoxide, $\mathrm{Nb}_{2} \mathrm{O}_{5}$ (Sigma-Adrich Chemistry, 99.9\% purity).

\subsection{Methods of preparation and characterization}

The initial mixtures for $5 \mathrm{YSZ}$ were $1: 4$ ratio of $20 \mathrm{wt} \% \mathrm{YSZ}$ and $80 \mathrm{wt} \% \mathrm{Al}_{2} \mathrm{O}_{3}$ respectively. These compositions was mixed with an addition of the varied amount of niobium pentoxide ( $0 \mathrm{wt} \%, 0.3 \mathrm{wt} \%, 0.5 \mathrm{wt} \%$ and $0.7 \mathrm{wt} \%$ ). The weighted powders were mixed by using a conventional mortar and pestle for about 30 minutes on each composition and ethanol was added to facilitate the mixing process. The homogenized powder werecalcined at $1000^{\circ} \mathrm{C}$ for 2 hours with rate of $10^{\circ} \mathrm{C} \mathrm{min}^{-1}$ followed by crushing the dried samples using mortar and pestle. Each of the compositions was hydraulically pressed at 220 $\mathrm{MPa}$ into a $20 \mathrm{~mm}$ diameter mould cavity and $3 \mathrm{~mm}$ thickness pellet. All the sintered pellets were sintered at $1650^{\circ} \mathrm{C}$ for 2 hours at heating rate of $10^{\circ} \mathrm{C} \mathrm{min}^{-1}$.

Differential thermal analysis (DTA) was used to detect the decomposition reaction especially on the heat absorption while thermal gravimetric analysis (TGA) is to measure the weight change in the tested samples as function of time or temperature. DTA-TGA was performed at $1500^{\circ} \mathrm{C}$ with heating rate of $10^{\circ} \mathrm{C} \min ^{-1}$ by using Pelkin Elmer 
thermoanalyzer. The different phases of samples were developed by using X-ray diffraction analysis (XRD) with $\mathrm{Cu} \mathrm{K} \alpha$ monochromatic radiation (XRD 6000, SHIMADZU). X-ray diffraction data for qualitative and Rietveld analysis were collected on the $2 \theta$ ranges from $5^{\circ}$ to $90^{\circ}$ with scan axis of $1 \% \mathrm{~min}$ and a step scan of $7 \mathrm{~s}$. The X-ray tube was operated at 40 $\mathrm{kV}$ and $50 \mathrm{~mA}$.

Vickers hardness values were taken by using Vickers Hardness Tester model of Mitutoyo HM-210/810-403E Japan by indentions technique at $1 \mathrm{~kg}$ load for 15 seconds on the sintered body. Vickers hardness values were measured by averaging 5 different readings on each of the sintered body. Bulk density of the sintered samples was calculated by using Archimedes principle and Pycnometer (AccuPcy 1330) with helped of Helium Pcynometer. All samples were coated with platinum (pt) by using sputter coater (JFC1600). Scanning electron microscopy was carried out to observe the morphology of the sintered ceramics samples by using SEM (JSM-6460LA, JEOL) and assisted with an energy dispersive X-ray (EDX).

\section{Results and discussion}

\subsection{DTA-TGA}

Thermal analysis appraises the behaviour of the sample under controlled temperature changes. TGA analysis was performed to understand the decomposition and measure the weight changes in ceramics materials over temperature and time. It is important to understand the thermal treatment and time, as it will effect or influence the ceramics composite materials such as degree of particle agglomeration and second phase size distribution.

Fig. 1 shows the behaviour for all ZTA samples, which can be seen on the TG curve patterns. The TG curve increase, which indicate that the weight gain from the beginning until the end of the tests done. A linear graph of weight gain respective to temperature trend can be seen at room temperature up to temperature of $580^{\circ} \mathrm{C}$. This shown that the weight increases gradually as temperature increases. The weight gained in the TG curve may be due to the oxidation of the ZTA samples in the air atmosphere at the beginning of the analyses. Increment of ZTA samples weight was ZTA, ZTA 0.3 , ZTA 0.5 and ZTA 0.7 with $0.14 \mathrm{mg}, 0.15 \mathrm{mg}$, and $0.165 \mathrm{mg}$. A slightly weight gain rate shown at temperature above $1420^{\circ} \mathrm{C}$ for all ceramics powder may be due to the oxidation reaction. 


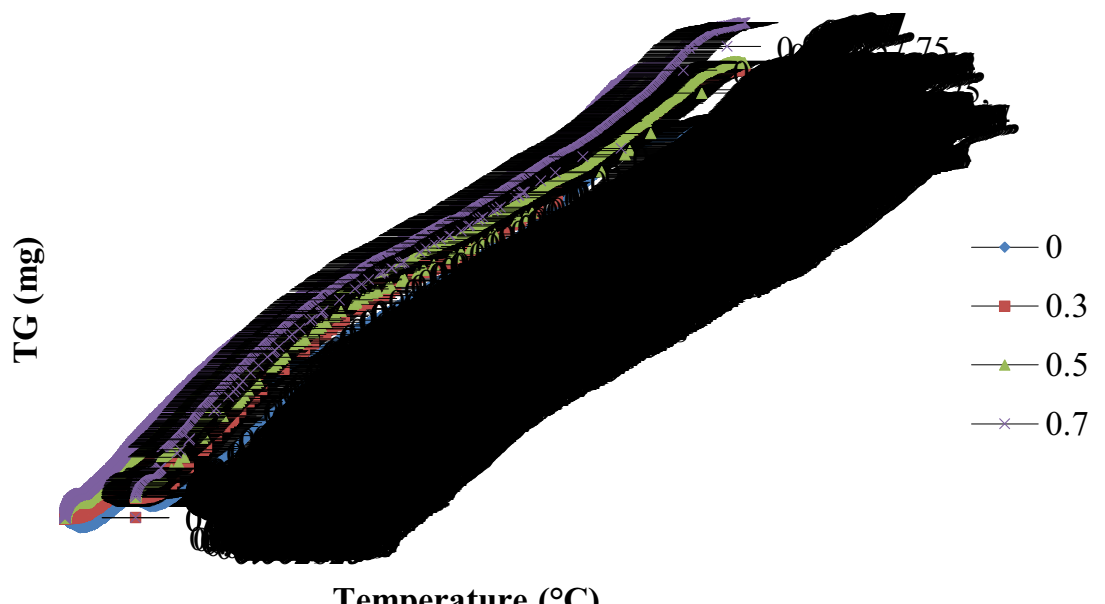

Temperature $\left({ }^{\circ} \mathrm{C}\right)$

Fig. 1. TGA curve for different ZTA composition.

Fig. 2 shows DTA curve shift from the exothermic peak to the endothermic peak for all ZTA compositions at about $1100{ }^{\circ} \mathrm{C}$ endothermic peak onset. A small difference between undoped and doped ZTA samples, which is exothermic peak at temperature of $185^{\circ} \mathrm{C}$ while the doped compositions, is at $210^{\circ} \mathrm{C}$. This shows that the ZTA powder for undoped ZTA undergo crystallization earlier compared to the doped sample. The shifted from exothermic to endothermic took place at temperature around $1100{ }^{\circ} \mathrm{C}$ and the endothermic peak for ZTA and ZTA0.7 was $1210^{\circ} \mathrm{C}$ while broaden endothermic peak for ZTA0.3 and ZTA0.5 powder was at temperature of $1280^{\circ} \mathrm{C}$. A small decrement in weight which about 0.0952 $\mathrm{mg}$ to $0.0951 \mathrm{mg}$ at temperature about $1030^{\circ} \mathrm{C}$. This indicates that this is the onset of the endothermic for the undoped ZTA samples to melts. The same trend could be seen at temperature range of $950^{\circ} \mathrm{C}$ to $960^{\circ} \mathrm{C}$ in which the weight loss from $0.101 \mathrm{mg}$ to $0.100 \mathrm{mg}$ for ZTA0.3 ceramics sample. The change of the exothermic to endothermic is due to the high temperature.

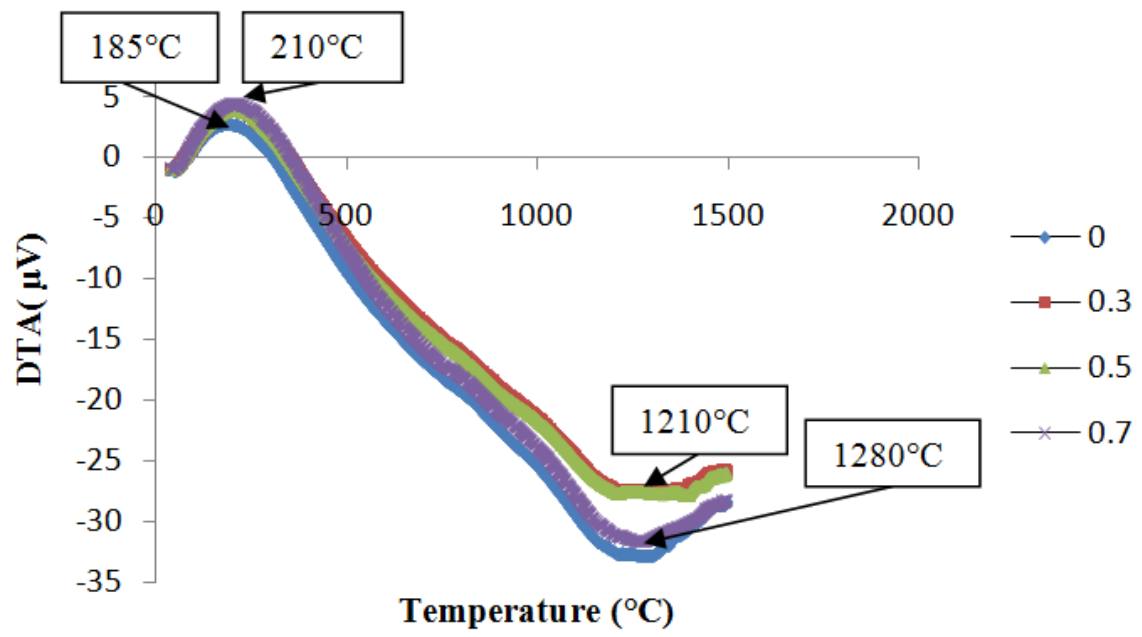

Fig. 2. DTA curve for different ZTA composition. 


\section{$3.2 \mathrm{XRD}$}

$\mathrm{X}$-ray diffraction (XRD) patterns of undoped and doped ZTA sintered body at $1650^{\circ} \mathrm{C}$ is shown in Fig. 2 and it can be observed that alumina phases present as dominant phase compared to tetragonal zirconia $\left(\mathrm{t}-\mathrm{ZrO}_{2}\right)$ and monoclinic zirconia $\left(\mathrm{m}-\mathrm{ZrO}_{2}\right)$. This is due to the $4: 1$ of alumina and YSZ used in each of the composition.

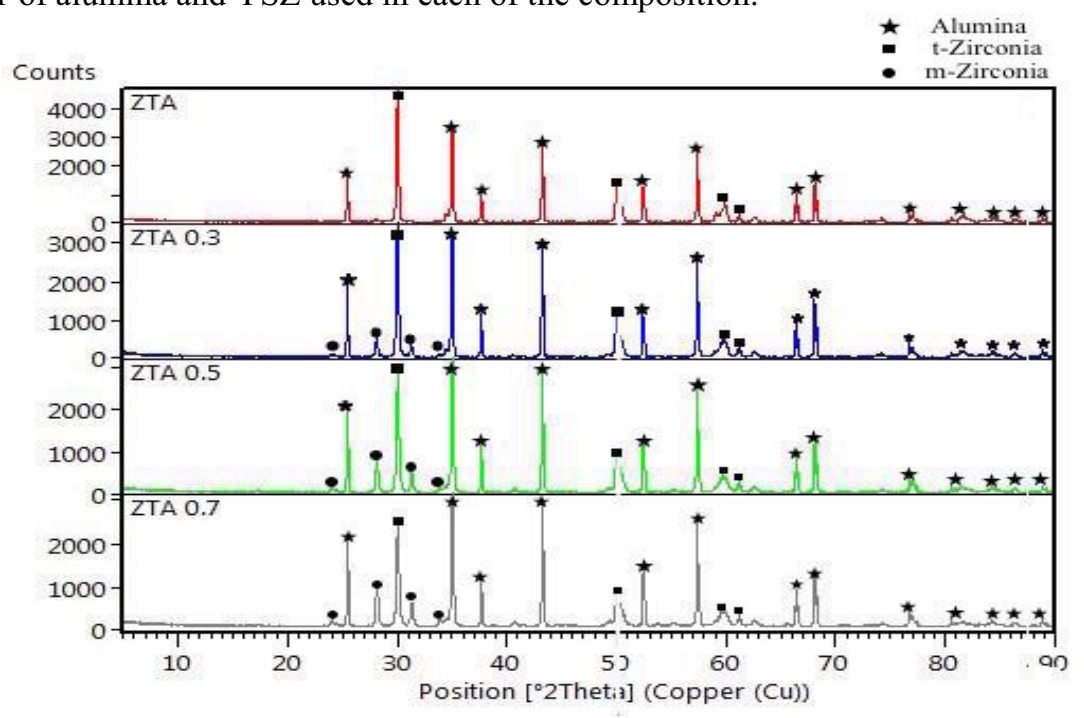

Fig. 3. XRD pattern for undoped and doped ZTA samples at $1650^{\circ} \mathrm{C}$.

For the pure ZTA sample, alumina present as major phase followed by $\mathrm{t}-\mathrm{ZrO}_{2}$ phase while for the niobium oxide doped ZTA samples, it can be seen that at all of the doped ZTA sample, alumina phases present beside the $\mathrm{t}-\mathrm{ZrO}_{2}$ (strongest peak) and both of the phases are surrounded by the traces peak of $\mathrm{m}-\mathrm{ZrO}_{2}$ at ranges of 20 to 40 position. Hassan et al. studied on the pentavalent oxide materials doped ZTA stated that alumina phases existed as the major phases with the tetragonal phase zirconia and a minor amount of monoclinic zirconia phases in all samples [2]. In addition, Shen et al. also revealed that $\mathrm{m}$ $\mathrm{ZrO}_{2}$ formed with major $\mathrm{t}-\mathrm{ZrO}{ }_{2}$ by using pentavalent oxide $\left(\mathrm{Ta}^{+}\right)$as dopant [10].

Besides, it is also have few noticeable changes on $\mathrm{m}-\mathrm{ZrO}_{2}$ peak intensity are increasing from pure ZTA up to $0.7 \mathrm{wt} \% \mathrm{Nb}_{2} \mathrm{O}_{5}$-doped $\mathrm{ZTA}$. The $\mathrm{t}-\mathrm{ZrO}_{2}$ phases present from pure ZTA to $0.7 \mathrm{wt} \%$ dopant added causing the tetragonal phase of zirconia peak intensity slightly decreasing. The same agreement pattern shown by pentavalent oxide doped ZTA in the XRD result of Hassan et al.

\subsection{SEM}

Fig. 4 (a), (b), (c) and (d) represents the morphology of SEM images for undoped and doped ZTA samples after being sintered at $1650^{\circ} \mathrm{C}$ for $2 \mathrm{~h}$ respectively. The figures show alumina grain with dissimilar grain sizes. Zirconia grains commonly appear in rounded and small in grain sizes which can be found in the intergranular position and the grain growth of the alumina grain is mainly due to the grain coalescence or fused that is correlated with the grain boundary migration $[2,11]$. Fang also stated that coalescence is the other grain growth mechanism, which related to the rapid grain growth [11]. 

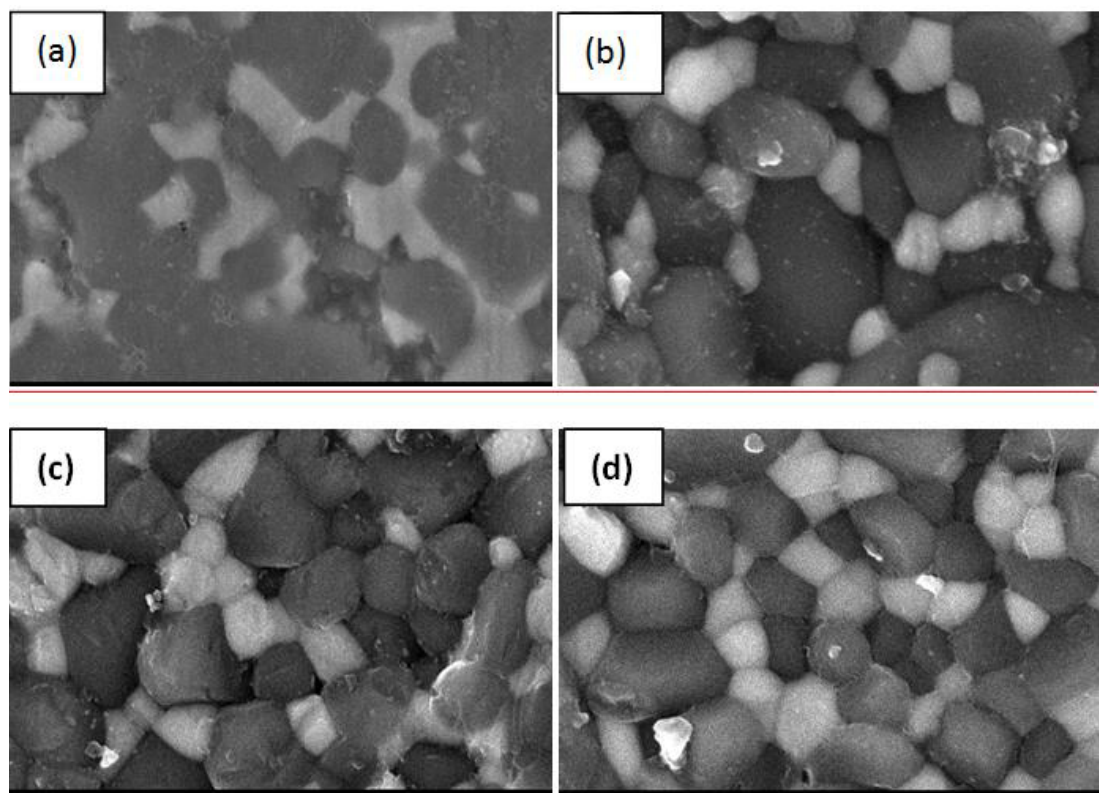

Fig. 4. SEM micrographs of undoped ZTA samples (a), ZTA0.3 (b), ZTA0.5 (c) and ZTA0.7 (d) at 10000x magnification.

In Fig. 4 (b), (c) and (d), the microstructure of doped ZTA samples distribution shows the same allocation as the undoped ZTA sample even with presence of niobium pentoxide particles. This is because the addition of $\mathrm{Nb}_{2} \mathrm{O}_{5}$ does not change the microstructure that is only up to $6 \mathrm{wt} \%$ niobium pentoxide addition $[12,13]$. Lesser porosity with good particle distribution and sizes shown by ZTA0.5 and ZTA0.7 compared to pure ZTA sample and $0.3 \mathrm{wt} \% \mathrm{Nb}_{2} \mathrm{O}_{5}$ dopant. This behaviour is may be due to the The small dopant agglomeration of $\mathrm{Nb}_{2} \mathrm{O}_{5}$ between the grain boundaries in the doped ZTA samples which may cause the particle shape of the ZTA0.3 and ZTA0.7 tend to be rounded shapes. It also due to the increasing amount of nobiumpentoxide as dopant.

\subsection{Density}

The bulk density for each of the compositions is tabulated on the Fig. 5. a correlation on the bulk density has improved by addition of $0 \mathrm{wt} \%$ up to $0.5 \mathrm{wt} \%$ of niobium pentoxide and decreases with the addition of $0.7 \mathrm{wt} \% \mathrm{Nb}_{2} \mathrm{O}_{5}$. The graph has illustrated the relation between the bulk density and the Vickers hardness gained. This trend resulted from the particle size of ZTA0.7 which is higher compared to the other composition after the synthesis method. Somton Et al stated that smaller particle size resulted to the low amount of porosity and higher apparent density [14]. Besides, broaden distribution of size will cause the densification with lesser shrinkage compared to the less range size distribution $[15,16]$. 


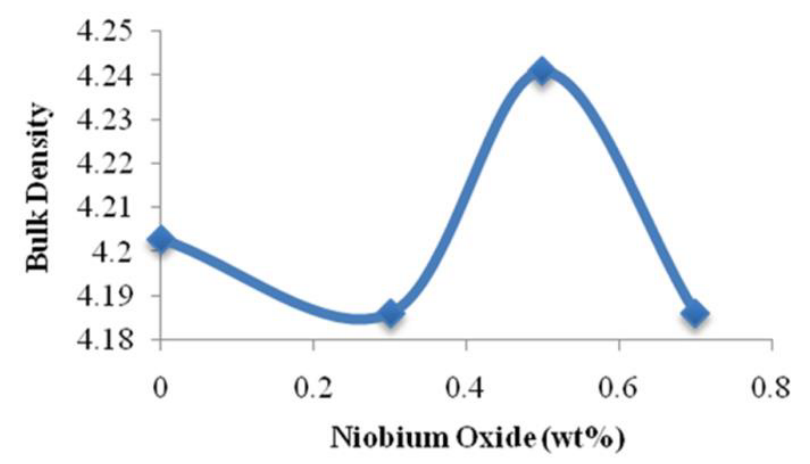

Fig. 5. Bulk Density of ZTA doped $\mathrm{Nb}_{2} \mathrm{O}_{5}$ composition.

\subsection{Vickers hardness}

Fig. 6 shows the resulted of the Vickers hardness, which plotted as function of dopant contents. Fig. 6 revealed that the Vickers hardness of ZTA undoped and doped niobium oxide increases with the dopant amount. But, this trend only could be seen up to $0.5 \mathrm{wt} \%$ of $\mathrm{Nb}_{2} \mathrm{O}_{5}$ content. The decreasing pattern presented from $0.5 \mathrm{wt} \%$ to $0.7 \mathrm{wt} \%$ addition of niobium oxide.

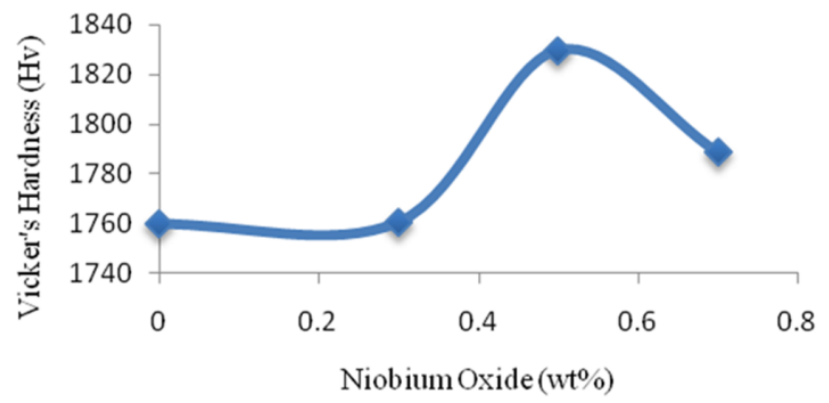

Fig. 6. Hardness obtained at different composition of ZTA.

The Vickers hardness of ZTA of undoped and doped niobium oxide is gradually increases from $1759.8 \mathrm{Hv}$ (ZTA) to the highest value of $1828.62 \mathrm{Hv}$ (ZTA0.5). This resulted of about $3.91 \%$ improvement compared to the undoped sample. The result also showed the slightly increased in hardness value from ZTA to ZTA0.3, which showed an increment of hardness about $0.8 \mathrm{Hv}$. While ZTA0.7, recorded a drop in hardness value from $1828.62 \mathrm{Hv}$ (ZTA0.5) to $1788.6 \mathrm{Hv}$ (ZTA0.7). Addition of more than $0.5 \mathrm{wt} \%$ niobium oxide would slightly decrease the hardness properties. This improvement is contributed by the highest densification and lowest porosity of ZTA0.5 samples. This improvement also was achieved with the presence of lowest apparent porosity and highest bulk density in ZTA0.5 sample compared to the other respective ZTA compositions. Zhang et al. mentioned that the different amounts of porosity in different particular sample could cause different hardness result and this might be contributed by sintering temperature used to eliminate porosity in the particular sample [17]. Porosity will give a great effect on the strength of the ceramics samples. Ceramics sample will be either failure in brittle manner or successfully survived from the stress applied. Rejab et al. stated that the size, shape and amount of pores in the ceramics samples would affect the strength as the cross sectional area over load lesser and caused the lower stress can supported [18]. He also added that the 
improvement of Vickers hardness of ZTA doped rare earth materials is because of the higher densification of the ceramics materials. It should be noted that the porosity could reduce the strength by formation of crack when it reach critical value and ceramics materials might fail without plastic deformation, so crack formed in the sample will propagate until it meet failure [2]. Therefore, it can be concluded that the addition of niobium oxide will significantly increase the hardness of the ZTA samples.

\section{Conclusions}

This research demonstrates the effect of $\mathrm{Nb}_{2} \mathrm{O}_{5}$ particles as dopant on the microstructure and mechanical properties of ZTA ceramics composites. It can be concluded that the addition of niobium pentoxide particles as dopant enhance the densification at $1650^{\circ} \mathrm{C}$ sintering temperature. The Vickers hardness increases from pure ZTA to $0.5 \mathrm{wt} \%$ of niobium pentoxide addition with the highest value of $1828.62 \mathrm{Hv}$ and then at $0.7 \mathrm{wt} \%$ $\mathrm{Nb}_{2} \mathrm{O}_{5}$ addition, it caused slightly decrease on the Vickers hardness. Bulk density and Vickers hardness shows a positive relation as it shown same trend. According to scanning electron microscopy, the porosity for the ZTA0.5 is slightly lesser compared to the undoped and other doped ZTA composition.

\section{References}

1. Campbell, C. Flake, Lighweight Materials - Understanding the basics (ASM International, USA, 2012)

2. A.M. Hassan, S.M. Naga, M. Awaad, Int. J. Refract. Metals Hard Mater., 48, 338 (2015)

3. M. Vallet-Regi, Bio-Ceramics with Clinical Applications (John Wiley \& Sons, West Sussex, 2014)

4. B. Basu, D.S. Katti, A. Kumar, Advanced Biomaterials: Fundamentals, Processing and Applications (John Wiley \& Sons, USA, 2010)

5. Nevarez-Rascon, A. Aguilar-Elguezabal, E. Orrantia, M.H. Bocanegra-Bernal, Int. J. Refract. Metals Hard. Mater., 27, 962 (2009)

6. V. Tebaldo, G. Gautier, Ceram. Int., 39, 2683 (2013)

7. Nevarez-Rascon, A. Aguilar-Elguezabal, E. Orrantia, M.H. Bocanegra-Bernal, Int. J. Refract. Metals Hard. Mater., 29, 333 (2011)

8. R. Hannink, P. Kelly, B. Muddle, J. Am. Ceram. Soc., 83, 461 (2000)

9. S. M. Kurtz, S. Kocagoz, C. Arnholt, R. Huet, M. Ueno, W.L. Walter, J. Mech. Behav. Biomed. Mater., 31, 107 (2014)

10. Y. Shen, R. Leckie, C. Levi, D. Clarke, ActaMater., 58, 4424 (2010)

11. Z. Fang, Sintering of advanced materials, (Woodhead Pub., Cambridge, 2010)

12. S. Vidyavathy, V. Kamaraj, Modern Applied Science, 3, 102 (2009)

13. D. Almeida, C. Cairo, C. Silva, M. Nono, Journal of Aerospace Technology and Management, 2, 195 (2010)

14. K. Somton, P. Sanguanwit, K. Sujirote, R. McCuiston, Journal of Metals, Materials and Minerals, 20, 1 (2010)

15. T. Yeh, M. Sacks, J. Am. Ceram. Soc., 71, 841 (1988)

16. B. Lawn, D. Marshall, , J. Am. Ceram. Soc., 62, 347 (1979)

17. D. Zhang, T. Yoshida, T. Oekermann, K. Furuta, H. Minoura, Adv. Funct. Mater., 16, $1228(2006)$

18. N. Rejab, A. Azhar, M. Ratnam, Z. Ahmad, Int. J. Refract. Metals Hard. Mater., 36, 162 (2013) 